\title{
Evidence for Multiple Causality in the Development of Diabetic Hypertriglyceridaemia
}

\author{
D. Weiland, C. E. Mondon, and G. M. Reaven \\ Department of Medicine, Stanford University School of Medicine and Geriatric Research, Education and Clinical Center, \\ Veterans Administration Medical Center, Palo Alto, California, USA
}

Summary. The relationship between varying degrees of insulin deficiency and hypertriglyceridaemia in rats have been examined. Rats were studied 7-10 days after injection with streptozotocin, and plasma glucose concentrations used to classify rats as having either moderate $(200-350 \mathrm{mg} / \mathrm{dl})$ or severe diabetes (>350 mg/dl). A 2- to 3 -fold rise in plasma triglyceride (TG) concentration developed in six week old insulin deficient rats associated with elevated plasma non esterified fatty acid (NEFA) concentrations and decreased very low density lipoprotein secretion. Perfused livers from six week old rats with either moderate or severe diabetes were incapable of increasing hepatic TG secretion when perfusate NEFA concentrations were raised from 0.4 to $1.8 \mathrm{mmol} / \mathrm{l}$. In one year old, spontaneously obese rats, an equivalent degree of hypertriglyceridaemia could be produced with a lesser degree of insulin deficiency, and in this instance very low density lipoprotein secretion was increased over control values. Hepatic TG secretion by perfused livers from these rats with moderate diabetes approximately doubled when perfusate NEFA concentration was raised from 0.40 to $0.85 \mathrm{mmol} / 1$. These results emphasize the complex causality of diabetic hypertriglyeridaemia in situations characterised by comparable degrees of fasting hyperglycaemia.

Key words: Diabetes, insulin deficiency, hypertriglyceridaemia, very low density liporotein, hepatic perfusion, triglycerides.

Albrink has characterised hypertriglyceridaemia as "the hyperlipemia par excellence of the diabetic" [1]. There is, however, considerable disagreement con- cerning the pathogenesis of hypertriglyceridaemia in patients with diabetes. The controversy is particularly applicable to patients with significant fasting hyperglycaemia. Early suggestions were that hypertriglyceridaemia in this situation was due to a defect in lipoprotein removal from the plasma [2-4], presumably secondary to a decrease in lipoprotein lipase (LPL) activity [2-6]. This hypothesis has received support from studies of the effect of chronic insulin deficiency on triglyceride (TG) metabolism in the rat [7-10] and the dog [11], in which hypertriglyceridaemia was associated with either a decrease in LPL activity $[7,8]$, or the rate of entry of very low density lipoprotein (VLDL)-TG into plasma [9-11].

Recent measurements of VLDL-TG kinetics in diabetic man are in contrast to these findings, and VLDL-TG secretion rates have been found to be elevated in the majority of adult onset diabetics with significant fasting hyperglycaemia $[1,14]$. Thus, direct measurements suggest that VLDL-TG secretion is increased, not decreased, in diabetic patients with significant fasting hyperglycaemia. The current experiments were undertaken in an effort to explore some of the reasons for the discrepancies and to see if these apparently dichotomous positions as to the cause of diabetic hypertriglyceridaemia in subjects with significant fasting hyperglycaemia could be reconciled.

\section{Methods}

\section{Animals}

All experiments were performed on male Sprague-Dawley rats. Two groups of rats were used for these studies: one group of rats were 5-6 weeks old, weighing $150-200 \mathrm{~g}$, while the other group were one year of age, and weighed more than $600 \mathrm{~g}$. Insulin-deficient diabetes was induced by intravenous injections of strep- 
tozotocin (Upjohn Co., Lot $\# 60140$ ), diluted in $0.01 \mathrm{~mol} / \mathrm{l}$ citrate buffer ( $\mathrm{pH} 4.5$ ). Varying doses of streptozotocin were injected $(22-50 \mathrm{mg} / \mathrm{kg}$ body weight) in order to produce desired combinations of hyperglycaemia and hypoinsulinaemia. Control rats were simultaneously injected with citrate buffer solution. Rats were maintained on a 12 hour dark $(1800 \mathrm{~h}-0600 \mathrm{~h})$-light $(0600 \mathrm{~h}-1800 \mathrm{~h})$ cycle, and allowed free access to standard rat chow (Wayne Lab Blox). Experiments were carried out 7-10 days after the injection of either streptozotocin or the citrate buffer solution.

\section{Plasma Glucose, Insulin, Triglyceride (TG) and Free Fatty Acid (FFA) Concentrations}

Food was removed at $0800 \mathrm{~h}$ and blood obtained from the tail vein for measurement of plasma glucose [15], insulin [16], TG [17], and NEFA [18] concentrations $6 \mathrm{~h}$ later. The blood was collected into tubes rinsed with $5 \%$ EDTA. Plasma was separated in a refrigerated centrifuge and stored frozen. Diabetic animals were divided into two groups on the basis of their plasma glucose concentrations: rats whose plasma glucose concentration were $200-350 \mathrm{mg}$ / $\mathrm{dl}$ were classified as having moderate diabetes, while rats whose plasma glucose concentrations exceeded $350 \mathrm{mg} / \mathrm{dl}$ were defined as having severe diabetes. Rats with moderate diabetes gained weight at the same rate as did control rats, while weight of rats with severe diabetes remained stable. Neither ketonuria nor ketonaemia were observed.

\section{Hepatic TG Secretion}

Hepatic TG secretion by livers from control and diabetic rats was determined by in situ perfusion techniques previously described [19-21]. All studies were started in the early afternoon, $6 \mathrm{~h}$ after food withdrawal. Both liver and blood donor animals were anaesthetized with sodium thiamylal $(6.2 \mathrm{mg} / 100 \mathrm{~g}$ body weight) prior to surgery. The perfusing medium consisted of $90 \%$ defibrinated rat blood, obtained from retired male breeding stock maintained on laboratory chow, and $10 \%$ Krebs Ringer bicarbonate buffer containing $3 \mathrm{~g} / 100 \mathrm{ml}$ of bovine serum albumin (hematocrit of $40 \%$ ). The perfusate volume at the start of recirculation averaged $42 \mathrm{ml}$ for studies of young rats, and $52 \mathrm{ml}$ for studies of old rats. Livers were perfused at constant flow rates, averaging $1 \mathrm{ml} / \mathrm{min} / \mathrm{g}$ liver from 0 to $120 \mathrm{~min}$. The perfusate also contained porcine insulin which was infused at rates of $10 \mathrm{mU} / \mathrm{h}$ for young rats and $13 \mathrm{mU} / \mathrm{h}$ for old rats in order to maintain perfusate insulin concentration at approximately $50 \mu / \mathrm{ml}$. In addition, each liver received continuous infusion $(2.3 \mathrm{ml} / \mathrm{h})$ of oleic acid complexed with rat serum solution prepared as previously described [21]. Three different NEFA concentrations $(18,36$ or $72 \mathrm{mmol} / \mathrm{l})$ were infused in order to attain NEFA concentrations of approximately $0.4,0.8$ and $1.9 \mathrm{mmol} / 1$. Perfusate samples of $1.8 \mathrm{ml}$ were withdrawn from the reservoir flask at $0,30,60,90$ and $120 \mathrm{~min}$. Hepatic TG secretion was calculated from net changes in perfusate TG concentration between 30 and $120 \mathrm{~min}$ after correcting for addition of the infusate solution and sampling losses.

\section{$V L D L-T G$ Secretion}

VLDL turnover rates were determined by following the rate of removal from the plasma of prelabelled VLDL-TG obtained from female donor rats $[10,21]$. Both control and diabetic rats were used as donors, and in all instances they were maintained on a fatfree diet for 4-7 days prior to being used. On the day of the experiment food was removed from donor rats at $0800 \mathrm{~h}$, and $4 \mathrm{~h}$ later they were injected via the tail vein under light ether anaes- thesia with $400 \mu \mathrm{Ci}$ of ${ }^{3} \mathrm{H}$-glycerol. The donor rats were exsanguinated under sodium thiamylal anaesthesia $20 \mathrm{~min}$ later. (Previous experiments had shown maximal incorporation of ${ }^{3} \mathrm{H}$-glycerol into TG 20 min after injection, at which time $95 \%$ of the lipid extractable radioactivity was incorporated into VLDL-TG as determined by ultracentrifugation and thin-layer chromatography.) The resultant plasma was then ultracentifuged, and aliquots of isolated VLDL, were injected into the experimental rats at $1400 \mathrm{~h}, 6 \mathrm{~h}$ after removal of food. After the administration of the labelled VLDL the tail was amputated proximal to the site of injection, and $0.4 \mathrm{ml}$ of blood was collected into capillary tubes rinsed with a 5\% EDTA solution $5,10,15$ and $20 \mathrm{~min}$ after the injection. These samples took 1 to $2 \mathrm{~min}$ to collect and in all subsequent calculations the time used for a collection period was the mean of the beginning and ending times of the blood collection. The plasma was separated by centrifugation and stored frozen until analysed. Samples were extracted with chloroform-methanol, polar lipid removed with silicic acid, and each sample evaporated to dryness. Radioactivity was measured by liquid scintillation counting (Beckman LS235 ), using a standard toluene scintillation mixture. The half-time $\left(\mathrm{t}_{\gamma_{2}}\right)$ of VLDL-TG removal was directly determined from these measurements by a least squares linear regression analysis. VLDLTG turnover rate was calculated from the following formula: VLDL-TG turnover rate $=\left(\ln 2 \div t_{1 / 2}\right) \times($ plasma TG concentration) $\times$ (plasma volume). Since these studies were carried out under steady state conditions, VLDL-TG turnover rate = VLDLTG removal rate $=$ VLDL-TG secretion rate. In this paper this measurement is referred to as VLDL-TG secretion rate.

Plasma volume of animals from each group was estimated by the standard dye dilution technique after the intravenous injection of Evans Blue, and the average plasma volume obtained by this method for each group was used in the above formula to calculate VLDL-TG secretion rate. Diabetes did not lead to a significant change in plasma volume in these animals. Results are expressed as mean $\pm S E M$, and the statistical significance between groups determined by Student's t-test.

\section{Results}

\section{Young Rats}

A. Plasma Glucose and Insulin Concentrations. Mean \pm SEM plasma glucose and insulin concentrations in 6 week old control and diabetic rats are shown in Table 1. The dose of streptozotocin needed to induce the desired degree of hyperglycaemia and hypoinsulinaemia varied somewhat from experiment to experiment, and not all rats reacted to a given dose of streptozotocin in a similar fashion. In general rats with moderate diabetes (fasting glucose 200$350 \mathrm{mg} / \mathrm{dl}$ ) received $35-45 \mathrm{mg}$ streptozotocin $/ \mathrm{kg}$ body weight, while it usually took $50-60 \mathrm{mg} / \mathrm{kg}$ body weight to produce severe diabetes (glucose > $350 \mathrm{mg} / \mathrm{dl}$ ). The degree of hyperglycaemia clearly varied directly with the degree of hypoinsulinaemia (Table 1).

B. Plasma TG and NEFA Concentrations. Plasma TG levels were higher than control values in young rats with moderate diabetes $(\mathrm{p}<0.01)($ Table 1$)$ and 
Table 1. Mean \pm SEM glucose, insulin, triglyceride and NEFA concentrations in the experimental groups

\begin{tabular}{|c|c|c|c|c|c|c|}
\hline \multirow[t]{2}{*}{ Age } & \multicolumn{2}{|l|}{ Experimental group } & \multicolumn{4}{|c|}{ Chemical determination } \\
\hline & Metabolic state & $\begin{array}{l}\text { Number of } \\
\text { animals }\end{array}$ & $\begin{array}{l}\text { Glucose } \\
(\mathrm{mg} / \mathrm{dl})\end{array}$ & $\begin{array}{l}\text { Insulin } \\
(\mu \mathrm{U} / \mathrm{ml})\end{array}$ & $\begin{array}{l}\text { Triglyceride } \\
(\mathrm{mg} / \mathrm{dl})\end{array}$ & $\begin{array}{l}\text { NEFA } \\
(\mathrm{mmol} / \mathrm{l})\end{array}$ \\
\hline 6 week & Control & 23 & $147 \pm 6$ & $35 \pm 2$ & $95 \pm 9$ & $0.27 \pm 0.04$ \\
\hline 6 week & Moderate diabetes & 15 & $247 \pm 13$ & $21 \pm 3$ & $151 \pm 12$ & $0.63 \pm 0.09$ \\
\hline 6 week & Severe diabetes & 12 & $470 \pm 40$ & $14 \pm 2$ & $198 \pm 13$ & $0.81 \pm 0.08$ \\
\hline 6 week & Control & 12 & $135 \pm 4$ & $32 \pm 3$ & $98 \pm 6$ & $0.25 \pm 0.03$ \\
\hline 1 year & Control & 14 & $147 \pm 3$ & $44 \pm 2$ & $134 \pm 9$ & $0.32 \pm 0.50$ \\
\hline 1 year & Moderate diabetes & 10 & $293 \pm 38$ & $29 \pm 2$ & $248 \pm 18$ & $0.73 \pm 0.09$ \\
\hline
\end{tabular}

Table 2. Relationship between perfusate NEFA concentration and hepatic VLDL-TG secretion rate

\begin{tabular}{lllll}
\hline Age & Metabolic state & $\begin{array}{l}\text { Number of } \\
\text { animals }\end{array}$ & $\begin{array}{l}\text { NEFA concentration } \\
(\mathrm{mmol} / \mathrm{l})\end{array}$ & $\begin{array}{l}\text { VLDL-TG secretion } \\
(\mathrm{mg} / \mathrm{g} \text { liver/h) }\end{array}$ \\
\hline 6 week & Control & 8 & 0.41 & $1.0 \pm 0.2$ \\
6 week & Control & 8 & 0.84 & $1.6 \pm 0.2$ \\
6 week & Control & 8 & 2.00 & $2.9 \pm 0.3$ \\
6 week & Moderate diabetes & 7 & 0.82 & $0.6 \pm 0.1$ \\
6 week & Moderate diabetes & 7 & 1.98 & $0.8 \pm 0.1$ \\
6 week & Severe diabetes & 6 & 0.79 & $0.4 \pm 0.1$ \\
6 week & Severe diabetes & 6 & 1.98 & $0.5 \pm 0.2$ \\
1 year & Control & 7 & 0.40 & $1.1 \pm 0.1$ \\
1 year & Control & 7 & 1.22 & $2.2 \pm 0.2$ \\
1 year & Moderate diabetes & & 0.85 & $1.7 \pm 0.2$ \\
\hline
\end{tabular}

still higher in rats with severe diabetes $(\mathrm{p}<0.05)$. Similar increments in NEFA levels were also found (Table 1).

C. Hepatic TG Secretion. Livers from control rats were perfused at three different perfusate NEFA concentrations $(0.41,0.84$, and $2.0 \mathrm{mmol} / \mathrm{l})$, each increment resulting in an increase in hepatic TG secretion (Table 2). In contrast, hepatic TG secretion by livers from moderately and severely diabetic rats increased only slightly when perfusate NEFA concentrations were increased to $1.98 \mathrm{mmol} / 1$. Hepatic TG secretion by livers from diabetic rats at high perfusate NEFA concentrations was less than that of controls at physiological NEFA levels $(0.41 \mathrm{mmol} / \mathrm{l})$.

D. VLDL-TG Secretion Rates. VLDL-TG secretion rates of the three experimental groups of young rats are shown in Figure 1. The highest secretion rates were found in control rats, and VLDL-TG secretion rates declined with increasing insulin dificiency. Comparable decreases in VLDL-TG secretion were found in the insulin deficient rats when VLDL was prepared from normal, moderate, or severely diabetic donors. An overall mean secretion rate for the 12 rats in each of the three experimental groups was therefore calculated. The mean ( \pm SEM) VLDL-TG secretion rate was $1.36 \pm 0.08 \mathrm{mg} / \mathrm{min}$ in young control rats, and this fell to $0.93 \pm 0.05$ and $0.76 \pm$ $0.04 \mathrm{mg} / \mathrm{min}$ in rats with moderate and severe diabetes, respectively.

\section{Old Rats}

A. Plasma Glucose and Insulin Concentrations. In order to induce hyperglycaemia of a magnitude similar to that seen in young rats, smaller doses of streptozotocin were needed in 12 month old rats. As with young rats, the effect of a specific dose of streptozotocin was variable. In general streptozotocin $20-30 \mathrm{mg} / \mathrm{kg}$ body weight was needed to produce plasma glucose levels of $200-350 \mathrm{mg} / \mathrm{dl}$.

The plasma glucose and insulin concentrations of old rats are shown in Table 1. Plasma glucose levels of old control rats were not significantly different from those of young controls, but plasma insulin levels were significantly $(\mathrm{p}<0.05)$ elevated in old as compared to young control rats. Plasma glucose values of old rats with moderate diabetes were significantly higher $(\mathrm{p}<0.01)$ than controls. Plasma insulin 


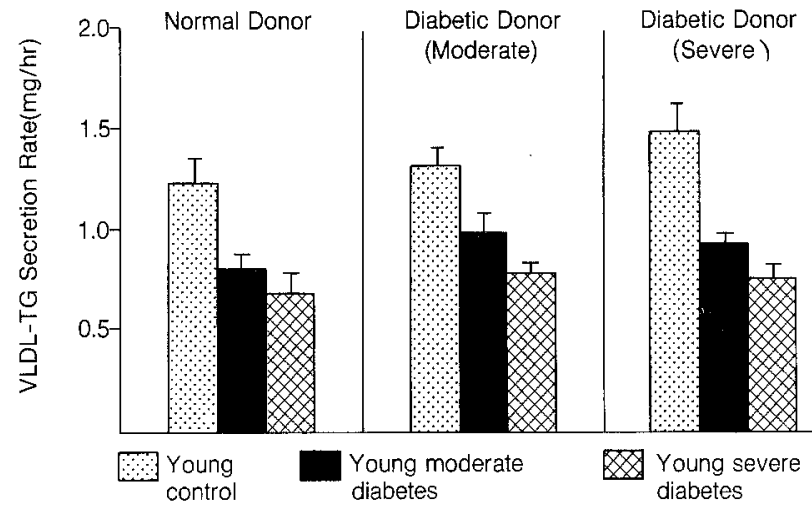

Fig. 1. Effect of varying degrees of insulin deficiency on VLDLTG secretion rates in six week old rats. VLDL-TG secretion was determined following the injection of prelabelled VLDL-TG from normal donors, and from donors with either moderate or severe diabetes. Prelabelled VLDL from each of the three types of donor rats was injected into control rats $(n=4)$, rats with moderate diabetes $(n=4)$, and rats with severe diabetes $(n=4)$

concentrations of old rats with moderate diabetes were lower $(\mathrm{p}<0.05)$ than that of old control rats, but were not statistically different from those of young control rats.

B. Plasma TG and FFA Concentration. Both plasma TG and NEFA concentrations were slightly, but significantly, higher $(\mathrm{p}<0.01)$ in old as compared to young control rats (Table 1 ). Moderate diabetes in old rats led to significant $(\mathrm{p}<0.01)$ elevations of both TG and NEFA concentrations when compared to old control rats. Furthermore, the TG and NEFA concentrations of the old control rats were comparable to those seen in young rats with a comparable degree of hyperglycaemia.

C. Hepatic TG Secretion. Hepatic TG secretion by perfused livers from both groups of old rats is shown in Table 2. Hepatic TG secretion by perfused livers of old control rats increased as perfusate NEFA concentrations were raised, as in young rats. The induction of moderate diabetes did not modify this relationship, and the VLDL-TG secretion rate from livers of old rats with moderate diabetes was comparable to that from livers of young control rats when perfused at comparable NEFA concentrations. Livers from old rats with moderate diabetes secrete more TG when they are perfused with elevated NEFA concentrations $(0.85 \mathrm{mmol} / \mathrm{l})$ higher than that obtained in vivo, than do livers of either young or old control rats when they are perfused at normal $(0.40 \mathrm{mmol} / \mathrm{l})$ in vivo NEFA concentrations.

D. VLDL-TG Secretion Rate. The in vivo VLDL-TG secretion rates of both groups of old rats were deter- mined following the injection of labelled VLDL-TG prepared from normal donor rats. VLDL-TG secretion of control rats $(\mathrm{n}=6)$ was $1.51 \pm 0.1 \mathrm{mg} / \mathrm{min}$ and rose to $2.1 \pm 0.1 \mathrm{mg} / \mathrm{min}$ in old rats with moderate diabetes. VLDL-TG secretion in old rats with moderate diabetes was significantly $(\mathrm{p}<0.01)$ greater than in young or old control rats.

\section{Discussion}

These results emphasise the universal nature of the development of hypertriglyceridaemia secondary to insulin deficiency. Streptozotocin-induced decrease in insulin secretion led to significant elevations of plasma TG in all the animals. Hypertriglyceridaemia occurred in both young and old rats, and was seen in the absence of dietary fat intake. The results also demonstrate the crucial role played by circulating insulin levels in the control of plasma TG levels.

On the other hand, the results also emphasise the complex causality of diabetic hypertriglyceridaemia. Streptozotocin was used to induce insulin deficiency and diabetes in rats of varying ages, and by adjusting the dose it was possible to create a variety of metabolic conditions. Although all of the experimental groups developed hypertriglyceridaemia, the reason for the rise in plasma TG concentrations was not the same in all groups. Hypertriglyceridaemia in young rats was associated with a decrease in VLDLTG secretion, indicating that insulin deprivation leads to hypertriglyceridaemia as the result of a defect in removal of VLDL-TG from plasma. As such, these results are in agreement with earlier studies of insulin deficient man [2-6], rat [7-10], and $\operatorname{dog}[11]$.

The situation in the older diabetic rats was clearly different. VLDL-TG secretion was increased in these animals, and seems to account for the development of hypertriglyceridaemia. Although the results of the current studies may not provide a definitive explanation for the apparent difference in the mechanism of diabetic hypertriglyceridaemia in young as compared to old rats, the data provide the basis for a reasonable hypothesis. In order to induce hyperglycaemia of comparable severity in young and old rats, it was necessary to give older rats less streptozotocin. This is at least partly due to insulin resistance in these old, spontaneously obese rats, and indirect evidence for this insulin resistance can be seen in Table 1. These results demonstrate that old control rats have significantly elevated basal plasma insulin levels as compared to young control rats, in spite of having comparable plasma glucose levels. This difference is accentuated in the postprandial state [22]. There is 
also experimental evidence that adipocytes isolated from such rats have fewer insulin receptors, as well as an intracellular defect in glucose oxidation $[23,24]$. As the result of this insulin resistance, a relatively moderate decrease in insulin secretion in old rats leads to significant hyperglycaemia, despite fasting insulin levels being comparable to those in normal young rats. Under these conditions it appears that the liver is capable of responding to increased levels of circulating NEFA by increasing hepatic TG synthesis and secretion. It should also be remembered that insulin deficiency leads to an increase in TG secretion by the intestine [10], and this phenomenon may also contribute to the increase in TG secretion by moderately diabetic old rats.

The differences in hepatic TG secretion by perfused liver from young and old rats almost certainly accounts for the disparate results noted in attempts to define the mechanism of hypertriglyceridaemia in man and animals [2-14] with comparable degrees of fasting hyperglycaemia. There are presumably two mechanisms for the development of diabetic hypertriglyceridaemia. Absolute insulin deficiency of a certain magnitude leads to hypertriglyceridaemia, which is secondary to a defect in VLDL-TG removal from plasma. In this situation the degree of insulin deficiency is so severe the liver is apparently incapable of responding to an increase in circulating NEFA levels by increasing hepatic TG synthesis and secretion. In contrast, hyperglycaemia of a comparable degree, which is due to a combination of relative insulin deficiency and insulin resistance, can lead to increased hepatic (and intestinal) VLDL-TG secretion, and hypertriglyceridaemia. This is presumably because circulating insulin levels in this situation are high enough to enable the liver to respond to elevated NEFA concentrations by increasing hepatic VLDLTG secretion. If this hypothesis is applied to the apparently conflicting observations [2-14] on the pathogenesis of diabetic hypertriglyceridaemia the controversy becomes more apparent than real. All of the available data are consistent with the idea that there are at least two causes of diabetic hypertriglyceridaemia and the mechanism responsible for hypertriglyceridaemia in a given diabetic patient is not a simple function of the magnitude of the hyperglycaemia.

It should be emphasised that the explanation for increased hepatic TG secretion in old diabetic rats is based upon the assumption that different tissues have disparate responses to similar circulating insulin levels. Thus, we are postulating that insulin levels in insulin-resistant old rats are too low to prevent accelerated lipolysis and increased plasma NEFA levels, while at the same time they are high enough to enable the liver to respond to the increased NEFA flux in a normal fashion. Although we have no direct evidence for this assumption in this situation, we think it reasonable for two reasons. First there is a precedent for this kind of phenomenon. Thus, previous studies of endogenous hypertriglyceridaemia in man have indicated that there can be resistance to peripheral glucose uptake at insulin levels which permit normal suppression of hepatic glucose output [25]. Furthermore, insulin concentrations in the portal vein of old diabetic rats were higher than those measured in the peripheral circulation, and this difference may have been sufficient to permit normal hepatic VLDL-TG synthesis and secretion to continue at a time when peripheral glucose metabolism was greatly impaired. These possibilities are open to experimental verification, and establishing the fact that they exist in old rats with diabetic hypertriglyceridaemia would certainly strengthen the hypothesis that we have offered to explain this phenomenon. Such efforts are currently in progress in our laboratory.

Acknowledgements. This work was supported in part by a grant from the National Institutes of Health, HL 20397, and from the Research Services of the Veterans Administration.

\section{References}

1. Albrink MJ (1974) Dietary and drug treatment of hyperlipidemia in diabetes. Diabetes 23: 913-918

2. Bierman EL, Bagdade J, Porte Jr D (1966) Concept of pathogenesis of diabetic lipemia. Trans Assoc Am Physicians 79: 348-360

3. Bagdade JD, Porte JrD, Bierman EL (1967) Diabetic lipemia: form of acquired fat induced lipemia. N Engl J Med 276: 427-433

4. Bagdade JD, Porte Jr D, Bierman EL (1968) Acute insulin withdrawal and the regulation of plasma triglyceride removal in diabetic subjects. Diabetes 17: 127-132

5. Pykälisto OJ, Smith PH, Brunzell JD (1975) Determination of human adipose tissue lipoprotein lipase: Effect of diabetes and obesity on basal and diet-induced activity. $\mathrm{J}$ Clin Invest 56: $1108-1117$

6. Nikkilä EA, Huttunen JK, Ehnholm C (1977) Postheparin plasma lipoprotein lipase and hepatic lipase in diabetes mellitus: relationship to plasma triglyceride metabolism. Diabetes 26: 11-21

7. Schnatz JD, Williams RH (1963) The effect of acute insulin deficiency in the rat on adipose tissue lipolytic activity and plasma lipids. Diabetes 12: 174-178

8. Kessler J I (1963) Effect of diabetes and insulin on the activity of myocardial and adipose tissue lipoprotein lipase of rats. $J$ Clin Invest 42: 362-367

9. Reaven EP, Reaven GM (1974) Mechanisms for development of diabetic hypertriglyceridemia in streptozotocin-treated rats. $\mathrm{J}$ Clin Invest 54: 1167-1178

10. Risser TR, Reaven GM, Reaven EP (1978) Intestinal very low density lipoprotein secretion in insulin-deficient rats. Diabetes 27: 902-908 
11. Basso L V, Havel R J (1970) Hepatic metabolism of free fatty acids in normal and diabetic dogs. J Clin Invest 49: 537-547

12. Nikkilä EA, Kekki M (1973) Plasma triglyceride transport kinetics in diabetes mellitus. Metabolism 22: 1-22

13. Kissebah A H, Adams PW, Wynn V (1974) Inter-relationship between insulin secretion and plasma free fatty acid and triglyceride transport kinetics in maturity onset diabetics and the effect of phenethylbiguanide (Phenformin), Diabetologia 10: $119-130$

14. Sigurdsson G, Nicoll A, Lewis B (1976) Metabolism of very low density lipoproteins in hyperlipidaemia: studies of apolipoprotein B kinetics in man. Eur J Clin Invest 6: 167-177

15. Kadish A H, Litle R L, Sternberg JC (1968) A new and rapid method for the determination of glucose by measurement of rate of oxygen consumption. Clin Chem 14: 116-131

16. Desbuquois B, Aurbach GD (1971) Use of polyethylene glycol to separate free and antibody bound peptide hormones in radioimmunoassays. J Clin Endocrinol Metab 33: 732-738

17. Wahlfeld A W (1974) Triglyceride determination after enzymatic hydrolysis. In: Bergmeyer $\mathrm{HU}$ (ed) Methods of enzymatic analysis, 2nd English ed. Academic Press, New York, p 1831-1835

18. Akio N, Okabe H, Kita M (1973) A new colorimetric microdetermination of free fatty acid in serum. Clin Chim Acta 43: 317-320

19. Mondon CE, Burton SD (1971) Factors modifying carbohydrate metabolism and effect of insulin in perfused rat liver. Am J Physiol 220: 724-734
20. Mondon CE, Burton SD, Ishida T (1968) Glucose tolerance and insulin response of potasssium deficient rat and isolated liver. Am J Physiol 215: 779-787

21. Gregg R, Mondon CE, Reaven EP, Reaven GM (1976) Effect of acute uremia on triglyceride kinetics in the rat. Metabolism 25: 1557-1565

22. Bracho-Romero E, Reaven GM (1977) Effect of age and weight on plasma glucose and insulin responses in the rat. $J$ Am Geriatr Soc 25: 299-302

23. Olefsky JM, Reaven GM (1975) Effects of age and obesity on insulin binding to isolated adipocytes. Endocrinology 96: 1486-1498

24. Olefsky JM (1976) The effects of spontaneous obesity on insulin binding, glucose transport, and glucose oxidation of isolated rat adipocytes. J Clin Invest 57: $842-851$

25. Bernstein RM, Davis BM, Olefsky JM, Reaven GM (1978) Hepatic insulin responsiveness in patients with endogenous hypertriglyceridaemia. Diabetologia 14: 249-253

Received: March 14, 1979 ,

and in revised form: November 13, 1979

Gerald M. Reaven, M. D.

Veterans Administration Medical Center (182 B)

3801 Miranda Avenue

Palo Alto, CA 94304

USA 